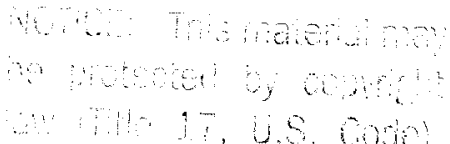

\title{
Optimal LGS pointing with a faint tip-tilt NGS
}

\author{
Richard Dekany \\ Caltech Optical Observatories, MS 11-17, Pasadena, CA 91125, USA
}

\begin{abstract}
Experience with the current generation of astronomical single laser guide star (LGS) adaptive optics ( $A O)$ systems has demonstrated system performance that is often limited by residual tip-tilt errors induced by the pancity of bright tip-tilt natural guide stars (NGS). To overcome this limitation, we are developing a new generation of tip-tilt sensors that will operate at near-infrared wavelengths where the NGS is sharpened to the diffraction limit. To optimize performance, single LGS $\Lambda O$ systems utilizing sharpened tip-tilt NGS should generally not point their LGS directly toward their science target. Rather, optimal performance for wide sky coverage is obtained by ofretting LGS pointing along a radius connecting the science larget and the tip-tilt NGS. We demonstrate that determination of the jointly optinized I, GS pointing angle and tip-tilt wavefront sensor (WFS) integration time can improve performance metrics by factors of several, particularly for faintest NGS operation. We find the I GS offset should be as much as $1 / 2$ the distance to the NGS to maximize Strohl matio at near-infrared wavelengths and $\approx 1 / 4$ the distane to the NGS to maximize ensquared energy, with lesser off-pointing for brighter NGS. Future $\Lambda \mathrm{O}$ systems may benefit from predictive determination of optimal I, GS oflsetting, based upon changing atmospheric conditions and observational geometries.
\end{abstract}

Keywords: 'Tip-tilt sensing, arlaptive optics, laser guide star, wavefront sensing

\section{INTRODUCTION}

Future laser guide star (LGS) adaptive optics (AO) systems developed for astronomical telescopes will increaningly bifurcate into two classes: wirle-ficld-of-view, partial-compensation $A O$ systens and narrow field-of-view, precision $A O$ systems. The former is rather tolerant of residual tip-tilt errors due to the relatively large dianeter point spread function (PSF) inherent in such systems. The latter, providing a PSF width approaching the limit set by photon diffraction, will demand the very best tip-tilt error compensation available' which cumently' requires optimal exploitation of available NGS.

Precision LGS AO science on telescopes of all sizes can benefit from improved tip-tilt error rejection. ${ }^{1}$ On small aperture telescopes, significant residual atmospheric tip-tilt measurement and bandwidth error and tilt anisoplanatism lead to large tip-tilt residuals. On the largest aperture telescopes, ${ }^{2}$ the benefits of large collecting area and finite outer scale significantly decrease tip-tilt wavefront oror energy, but these large structures are increasingly susceptible to new sources of error accentuated by exquisitely narrow PSF such as pointing jitter induced by the telescope drives, direct wind buffeting of the telescope structure, comelated segment wind shake, ${ }^{3}$ and transfer of wind buffeting on the dome to the telesenpe pier through surromending soil. ${ }^{1}$

Currently, LGS AO systems utilize visible-light NGS signal to determine tip-tilt errors in the science wavefront, typically because this optimizes light collecting efficiency for nedinfinared science and because low noise CCD or noiseless APD visible detector systems have been readily available. Unfortunately, the tip-tilt measuring performance using only partially-corrected visible PSF's results in rather large tip-tilt errors when guicling on the faintest NGS. To improve tip-tilt performance, we are developing a new generation of tip-tilt wavefront sensors that will enjoy improved measmement accuracy by operating at near-infrared wavelengths with low detector noise, where $\Lambda O$ correction to the diffraction-limited PSF width is routinely achieved. In return. we are driven to more complicated observing scenarios, typically involving the utilization of one or more near-infrared observing bands for tip-tilt wavefront sensing, while a different observing band is used simultaneously for scicnce observations.

Further author information: (Send correspondence to R.G.D.) R.G.D.: E-mail: rgd@astro.caltech.edu, Telephone: 16263956798

Adaptive Optics Systems II, edited by Brent L. Ellerbroek, Michael Hart, Norbert Hubin, Peter L. Wizinowich, Proc. of SPIE Vol. 7736,773625 - (C) 2010 SPIE - CCC code: 0277-786X/10/\$18 - doi: $10.1117 / 12.857841$

Proc. of SPIE Vol. 7736 773625-1 


\section{SCIENCE TARGET STREHL RATIO}

The science target Strehl ratio, $S_{S c a}$, is given by the product of the high-order wavefront error Strehl, $S_{H O}$, and the tip-tilt error Strehl, $S_{T}$,

$$
S_{S c i}=S_{H O} * S_{T}
$$

11. is worth noting that, the two terms contributing to science target Strehl ratio have different angular dependencies, described below, which is the fundanental driver that determines the optimal LGS pointing direction.

\subsection{High-order Wavefront Error Strehl Ratio}

For precision wavefront correction, the high-order wavefront error Strehl can be reasonably approximated via the Marechal approximation, ${ }^{5}$

$$
S_{H O}=e^{-\sigma_{H O}^{2}}
$$

where $\sigma_{H O}$ is the tip-tilt removed root-mean-square (RMS) residual wavefront error composed of various crror source terms, that can broadly be considered to contribute independently to an overall error variance,

$$
\sigma_{H O}^{2}=\sigma_{A A}^{2}+\sigma_{B}^{2}+\sigma_{F}^{2}+\sigma_{F A}^{2}+\sigma_{M}^{2}+\sigma_{O t h(T)}^{2}
$$

where $\sigma_{M}$, measurement error and $\sigma_{l}$, system temporal bandwidth error, arise from the need to collect wavefront information from the guide star of appropriate signal-to-noise ration (SNR) and the finite time reguired to process wavefront sensor information into deformahle mirror (DM) commands. All $\Lambda O$ systems will also sulfer from $\sigma_{F}$, fitting error, flue to finite correction spatial bandwidth. Single IGS AO systems will suffer $\sigma_{F A}$, focal anisoplanatism error (while multiple LGS AO systems suffer instead from residual tomography orror not considered here) Single conjugate $A O$ systems suffer from $\sigma_{A A}$, angular anisoplanatism between LCS pointing direction and science target, while multi-conjugate adaptive optics will suffer instean from gencralized anisoplanatism across the science field of view, again not considered in the scope of this study. Finally, $\sigma_{() t h e w}$ includes errors due to wavefront sensor aliasing, scintillation, chromatic errors, and any number of engineering implementation errors, such as DM hysteresis, WFS non-linearity, and wavefront calibration errors. In reality, the assumption of error independence somewhat overestimates the potential on-sky performance, but this secondorder cffect does not affect our basic conclusions here.

\subsubsection{Angular anisoplanatism error}

The high-order wavefront error due to angular anisoplanatism when a wavefront is measured from a clirection differing by angle $\theta$ from the direction of the science target is given by

$$
\sigma_{A A}=\left(\frac{\theta}{\theta_{0}}\right)^{5 /{ }^{j}}[\mathrm{rad} \text { of phase }]
$$

where $\theta_{0}$ is the isoplanatic angle which is a function of the $\frac{5}{3}$ vertical turbulence moment of the $C_{n}^{2}(h, t)$ profile. 6 


\subsubsection{Bandwidth and delay errors}

The residual high-order wavefront error due to finite temporal bandwidth and pure-rlelay errors is given by

$$
\sigma_{B}=\sqrt{\kappa\left(\frac{f_{G}}{f_{S}}\right)^{5 / 3}+28.4\left(T_{D e l a y} f_{G}\right)^{5 / 3}}[\text { rad of phase }]
$$

where $f_{G}$ is the Greenwood freculency, $f_{S}$ is the AO system correction servo bandwidth, and $\kappa$ is a coefficient that depends on the implementation of the servo control law ( $\kappa=1$ for a single-pole control law). ${ }^{7}$ For a single turbulent layer, where $f_{G}=0.127 v / r_{0}$, this can be simplified to be

$$
\sigma_{B}=\sqrt{\left(\frac{0.427 v}{r_{0} f_{S}}\right)^{5 / 3}+\left(\frac{0.427 v T_{D \cdot l a y}}{r_{0}}\right)^{5 / 3}}=\sqrt{35.7\left(\frac{v T_{I m}}{r_{0}}\right)^{5 / 3}+6.88\left(\frac{v T_{D r l u y}}{r_{0}}\right)^{5 / 3}}[\text { [rad of phase] }
$$

for an $\Lambda \mathrm{O}$ system whose servo bandwidth is inversely related to WFS integration time, $T_{l n t}$, via $f_{\mathrm{s}}=0.05 / T_{l m}$. a relationship typical of a well-designed controller.

The effective time delay for any clused-loop AO system is at least the integration time (1/2 the WFS integration time plus $1 / 2$ the I)M hold time). For now, we will assume $T_{D \text { del }}=2 T_{\text {lut }}$ and can thus summarize

$$
\sigma_{B}=7.58\left(\frac{v T_{l m l}}{r_{0}}\right)^{5 / 6}[\text { rad of phase }]
$$

\subsubsection{Fitting error}

The high-order wavefront cror due to finite spatial bandwidth of a wavefront corrector can be described as

$$
\sigma_{F}=\alpha\left(\frac{D}{\Delta x}\right)^{5 / 6}[\text { rad of phase }]
$$

where $\alpha$ is a coeflicient that depends on the corrector type and influcnes function, and $\Delta x$ is the corrector spacing, as projected onto the telescope prinary nirror. For our purpose here we wish to consider thin facesheet deformable mirrors ${ }^{7}$ having $a \approx 0.28$.

\subsubsection{Focal anisoplanatism error}

The high-order wavefront error due to focal anisoplanatism in a single LGS system is given by

$$
\left.\sigma_{F A}=\left(\frac{D}{d_{U}}\right)^{5 / 6} \text { [rad of phase }\right]
$$

where $d_{0}$ is the focal anisoplanatism coherence diancter which is a function of $\mu_{2}$, the second vertical moment of the $C_{n}^{2}(h, t)$ profile. 


\subsubsection{Measurement error}

The residual high-order wavefront error due to imperfect measurement can be described for a Shack-Hartmann wavefront sensor (WFS) as ${ }^{7}$

$$
\sigma_{M}=\frac{3 \pi d t}{16} \frac{w_{S p o t}}{\left(\frac{S}{N}\right)_{L G S}}[\mathrm{rad} \text { of phase }]
$$

where $w_{\text {Spot }}$ is the full-width at half-maximum (FWHM) of the guide star short exposure electronic PSF at the LGS WFS focal plane [radians of angle on sky], $\left(\frac{S}{N}\right)_{L G S}$ in the signal-to-noise ratio of the subaperture detection, $d$ is the subaperture diameter $d=D / N_{\text {Subap }}$ [meters], and $t$ is the error propagator for reconstruction, for which we will assmme a Fried geometry. ${ }^{7}$ Our model for subaperture inage width is given by,

$$
\begin{aligned}
& w_{\text {Spot }} \approx \sqrt{\left(\frac{\lambda_{\text {LGSS }} N_{\text {Subap }}}{D}\right)^{2}+\left(\frac{\lambda_{L G S}}{r_{0}}\right)^{2}+\sigma_{\text {Charge Diffusion }}^{2}} \\
& r \approx \sqrt{0.190+0.107 \ln N_{\text {Subap }}^{2}}
\end{aligned}
$$

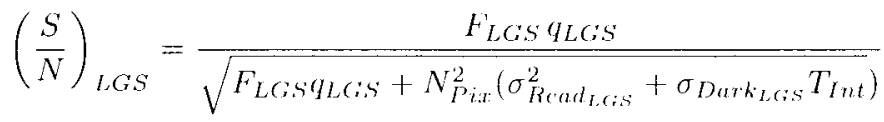

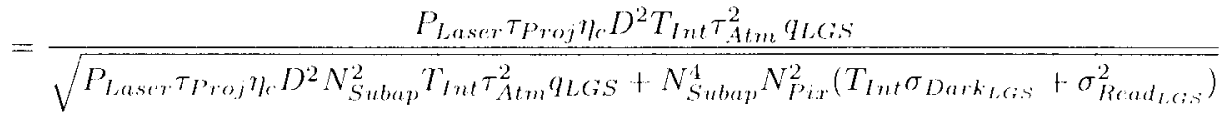

where $\lambda_{L G S}$ is the guide star laser wavelength, $N_{\text {Subap }}$ is the muber of wavefront sensor subapertures across

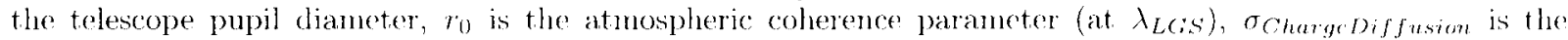
electronic broadening of the PSF after photodetection, qLGS is the quantmen efficioney of the LGS WFS detector. $N_{P i x}$ is the number of pixels across the diameter of a IGS WFS subaperture, $F_{L \text { cas }}$ is the laser return at the entrance pupil of the telescope $\left[p h m^{2} s^{1}\right], \eta$. is the laser return coupling efreicncy to the sodium layer. $\sigma_{\text {Real }}$ s;s is the root-medn-squared (RMS) readont noise of the LGS WFS [e--], $\sigma_{\text {Darkicss }}$ is the dark current variance in the LGS WFS [e- $\left.s^{-1}\right]$, PLase is the guide star laser power $[W]$ at the output of the laser itself. $\tau_{A t m}$ is the atmospheric transmission at LGS wavelength, $\tau_{P r o j}$ is the LGS beam transport and projector system transmission at LGS wavelength, $T_{I n t}$ is the WFS integration time.

\subsubsection{Other high-order wavefront errors}

Any practical $A O$ system will also suffer from a rmmber of smaller almospheric and implementation ferrors, such as WFS aliasing error, chromatic errors that arise when observing off-zonith, internal calibration errors, and non-common-path errors between $A O$ system and science instrument.

$$
\sigma_{\text {oun }}=\text { Constant }[\mathrm{rad} \text { of phase }]
$$

\subsection{Science Target Tip-tilt Error Strehl Ratio}

The science target Strehl ratio due to tip-tilt errors from (1) can be written ${ }^{8}$

$$
S_{T}=\frac{1}{1+\frac{\pi^{2}}{2}\left(\frac{\sigma_{T}}{\lambda_{S+i} / D}\right)^{2}}
$$

where $\sigma_{T}$ is the 1-axis RMS resiclual tilt error in the science wavefront, $\lambda_{S c i}$ is the science observing wavelength, and $D$ is the telescope entrance pupil diameter. We can expand by assuning independence of error terms to write 


$$
\sigma_{T}=\sqrt{\sigma_{T}^{2}+\sigma_{T B}^{2}+\sigma_{T A A}^{2}+\sigma_{T O t h e T}^{2}}
$$

where $\sigma_{T M}$ is the measurement error from the NGS-based tip-tilt measurement defined in analogy to (10) above, $\sigma_{T B}$ is the tip-tilt bandwidth error, and $\sigma_{T A A}$ is 1-axis tilt anisoplanatism, which corresponds to angular anisoplanatism for the tip-tilt wavefront modes.

\subsubsection{Tilt bandwidth error}

Residual science path tip-tilt crror clepends upon the latency of the tip-tilt measurement, as well as $L_{0}$, the outer scale of atmospheric turbulence. Under the frozen flow assumption.

$$
\sigma_{T B}=0.177\left(\frac{T_{T I n t}}{\tau_{0}}\right)\left(\frac{\lambda_{T i l t}}{D}\right)\left(\frac{r_{0}}{D}\right)^{\frac{1}{6}}[\operatorname{racl} \text { of phase }]
$$

where $T_{T \text { Int }}$ is the integration time in the tip-tilt sensor, $\lambda_{7}$ in is the tip-tilt sensing wavelength, $\tau_{0}$ is the atmospheric coherence time which depends upon atmospheric turbulence strength and wind speed, inchuling the effect of $L_{0}$, the outer scale of turbulence. ${ }^{7}$

\subsubsection{Tilt anisoplanatism error}

Tilt anisoplanatism arises when wavefrom tile measurements are made in a direction differing by angle 0 from the direction of the science target, and can be described for small angles by ${ }^{6}$

$$
\sigma_{T A A}=2.31 \sqrt{1-20.6\left(\frac{D}{L_{0}}\right)^{2}+27.4\left(\frac{D}{L_{0}}\right)^{7 / 3}} \sqrt{\frac{\partial^{2} \mu_{2}}{D^{7 / 3}}}[\text { [add of phase }]
$$

where $\mu_{2}$ is the second moment of the turbulence distribution ${ }^{7}$ and we have assumed the 1 -axis average of the rarlial and tangential tilt misoplanatism terms.

\subsection{Science Target Ensquared Energy}

The concentration of science light into either an inager or spectrograph detection element is appropriately described, for narrow spectral bandwidtl, by the ensquared energy (EE), which is lelated to the $\Lambda O$ system point spread function (PSF) by

$$
\mathrm{EF}=\int_{-s / 2}^{s / 2} \int_{s / 2}^{s / 2} \operatorname{PSF}(x, y) d x d y
$$

To investigate the noise properties of fast guiding on sharpened tip-tilt stars, we can approxinate the PSF in the regime of good $\Lambda O$ correction as the convolution of a diffraction-linited image core (approximated by a Gaussian function), containing a faction of energy corresponding to the Strehl ratio, and a blurring kernel that is also described by a Ganssian of width related to the two-dimensional residual tip-tilt crror

$$
P S F(x, y) \times S_{110} \exp \left(-\frac{x^{2}+y^{2}}{2(0.437 \lambda / D)^{2}}\right) \otimes \exp \left(-\frac{x^{2}+y^{2}}{2 \sigma_{1-D}^{2}}\right)
$$

Using (21), we can deternine the EE analytically based upon the residual tip-tilt error in the science target. wavefront, as determined in (17). 


\section{AO SYSTEM AND ATMOSPHERE MODELS}

We are motivated for this study to consider the potential performance of optinal J,GS pointing when used in combination with a near-infrared wavelength tip-tilt sensor to be developed for the workhorse Keck 2 LGS AO system ${ }^{3}$ at the W. M. Keck Observatory on Maund Kea. Our system will allow reconfiguration so that J and IIband science observations can be performed, while using K-band for tip-tilt sensing anywhere in the AO system field of regard, or alternatively $\mathrm{K}$-band science can be performed within a central 35 arcsecond dianeter field, with tip-tilt sensing in K-band utilizing NGS outside this central field. As the AO system high-order correction is improved over time, the potential also exists to utilize $H$ or J-bands for tip-tilt sensing.

To make concrete selections for our study, we adopt the parameters shown in Table 1 as specific to the Keck $2 \Lambda O$ system (as envisioned in the near future), where $\tau$ represents optical transmission, q quantum efficiency, and $\sigma_{\text {Rread }}$ and $\sigma_{\text {Dark }}$ the detector read noise and dark current noise as used in (14). The subscripts LGS refer to the high-order laser guide star wavefront sensor, while subscripts NGS correspond to the near-infrared NGS tip-tilt wavefront sensor.

Table 1. Basic AO system model parameter used in this paper. Deviations from these parameters are noted in the text when appropriate.

\begin{tabular}{|c|c|}
\hline Telescope Model Parameter & Value \\
\hline D) & $10 \mathrm{III}$ \\
\hline$N_{\text {Suluap }}$ & 20 \\
\hline$N_{P i t}$ & 4 \\
\hline$T_{P} r \omega j$ & 0.60 \\
\hline$\tau_{A t m}$ & 0.892 \\
\hline$T_{A O C H}$ & 0.85 \\
\hline qLCOS & 0.85 \\
\hline$\sigma_{\text {Charge Diffusion }}$ & 0.5 pixel FWHM \\
\hline$\tau_{T T S}$ & 0.85 \\
\hline qTTSS & 0.80 \\
\hline$\lambda_{L C S S}$ & $589 \mathrm{~nm}$ \\
\hline$\sigma_{\text {RradthS }}$ & $2.2 \mathrm{e}-\mathrm{RMS}$ \\
\hline$\sigma_{\text {Dartilis }}$ & $265 \mathrm{e} / \mathrm{sec} / \mathrm{pixel}$ \\
\hline$\sigma_{\text {RiradTTS }}$ & 3.5 e- RMS \\
\hline$\sigma_{\text {DarkTTS }}$ & $1 \mathrm{e}-/ \mathrm{sec} / \mathrm{pixel}$ \\
\hline$P_{\text {Lase't }}$ & $20 \mathrm{WCW}$ \\
\hline Mesospleric Na Return & 75 photons $\mathrm{cm}^{-2} \mathrm{~s}^{-1} \mathrm{~W}^{-1}$ \\
\hline$\sigma_{\text {Other }}$ & $90 \mathrm{~mm}$ RMS \\
\hline$\sigma_{\text {TTOther }}$ & 1.0 mas RMS \\
\hline IFS spaxel size (H-band) & 62 mats \\
\hline
\end{tabular}

\section{STREHL PERFORMANCE WITH LGS POINTING}

Having developed the expanded forms for (3) above, we can now proceed to study the behavior of LGS AO system performance in regimes linited by either residual high-order wavefront errors or by residual tip-tilt error terms. A convenient way to explore the continum of performance regimes is to consider the AO system science 
Table 2. Basic AO system model parameter used in this paper. Deviations from these parameters are noted in the text when appropriate.

\begin{tabular}{|l|l|}
\hline Atm Model Parameter & Value \\
\hline$r_{0}$ & $16 \mathrm{~cm}$ \\
\hline$\theta_{0}$ & $2.7 \mathrm{arcsec}$ \\
\hline$d_{0}$ & $4.28 \mathrm{ml}$ \\
\hline$\mu_{2}$ & $9.41 \times 10^{-6}$ \\
\hline$L_{0}$ & $30 \mathrm{ml}$ \\
\hline Turbulence spectrum & von Karman \\
\hline UTurbulence-woightecl & $9.5 \mathrm{~mm} \mathrm{~s}^{-1}$ \\
\hline Zcuith angle & $0 \mathrm{deg}$ \\
\hline
\end{tabular}

target performance as a function of the tip-tilt NGS brightness and off-axis distance, $\theta$. In the following, we will consider tip-tilt NGS located at $\theta<45$ " off-axis having near-infrared brightmesses $10<m_{H}\left(\right.$ or $\left.m_{K}\right)<17$.

Based on the performance model described above, and the AO) system and atmospheric parameters described in Tables 1 and 2, we calculate the contributions to science target Strehl ratio as a function of the

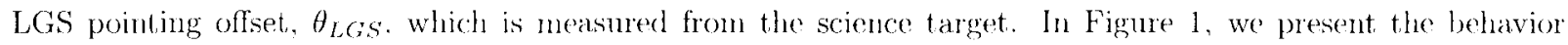
of $S_{H O}, S_{T}, S_{S c i}$, and $S_{N G S}$, the Strohl ratio of the tip-tilt NGS PSF. We see that the peak combined $S_{\text {Sre }}$ is obtained when the LGS is offset only slightly for a tip)-tilt. NGS 15" off-axis (due lo the relatively benign cffects of angular anisoplanatism on tip)-tilt. NGS Strehl and tilt anisoplanatism on science target Strehl.) When the tip-tilt NGS, however, is $30^{*}$ off-axis, a larger LGS pointing oflset is needed to obtain best science Strehl ratio, as this provides somewhat lower $\sigma_{T M}$ due to greater K-band $S_{N C}$ (though it is still just a few percent in the $\theta=30$ " case examined).
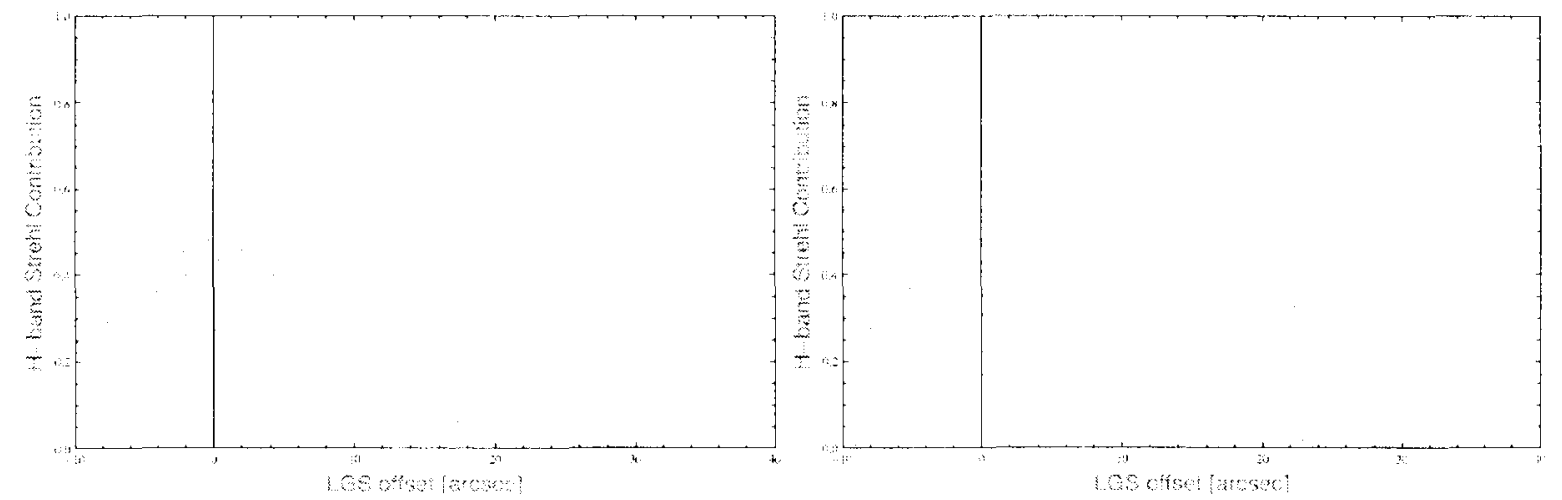

Figure 1. Science target H-band Strehl comtributions for a single LGS AO observation with $m_{K}=15$ tip-tilt NGS star located 15" (left) and 30" (right) from the science target. Within each figure, the tradeoff between high-order (solid) and tip-tilt (large dashed) Strehl ratio, total science Strehl ratio (dotted), and tip-tilt NGS Strehl ratio (dashed) is shown as $\theta_{L G S}$ is increased toward the tip-tilt NGS. In this model, only K-band photons are used in the tip-tilt NGS wavefront sensor, and the tip-tilt WFS integration time was held constant at 1 millisecond.

\subsection{Optimizing LGS Off-pointing and Tip-tilt WFS Integration Time}

To evaluate the potential gain of LGs off-pointing in a more realistic situation, we must consider the flexibility of the AO system operator to vary the tip-tilt NGS WFS integration time, $T_{T \text { Int }}$, to balance the SNR in the tip-tilt measurement term and the angular anisoplanatism error in the science target wavefront. 
Not surprisingly, the performance contours shown indicate that for any particular LGS pointing, there is an optimal choice of $T_{T I n t}$, with this optimal value moving to longer exposure times for decreasingly bright tip-tilt NGS. At the same time, the optimal value for $\theta_{L G S}=00_{L G S}^{O p t}$ moves to increasing angles for decreasing tip-tilt NGS brightness. This is because, as the tip-tilt measurement error grows with falling brightness, it is increasingly important to maintain the Strehl ratio of the tip-tilt NGS.
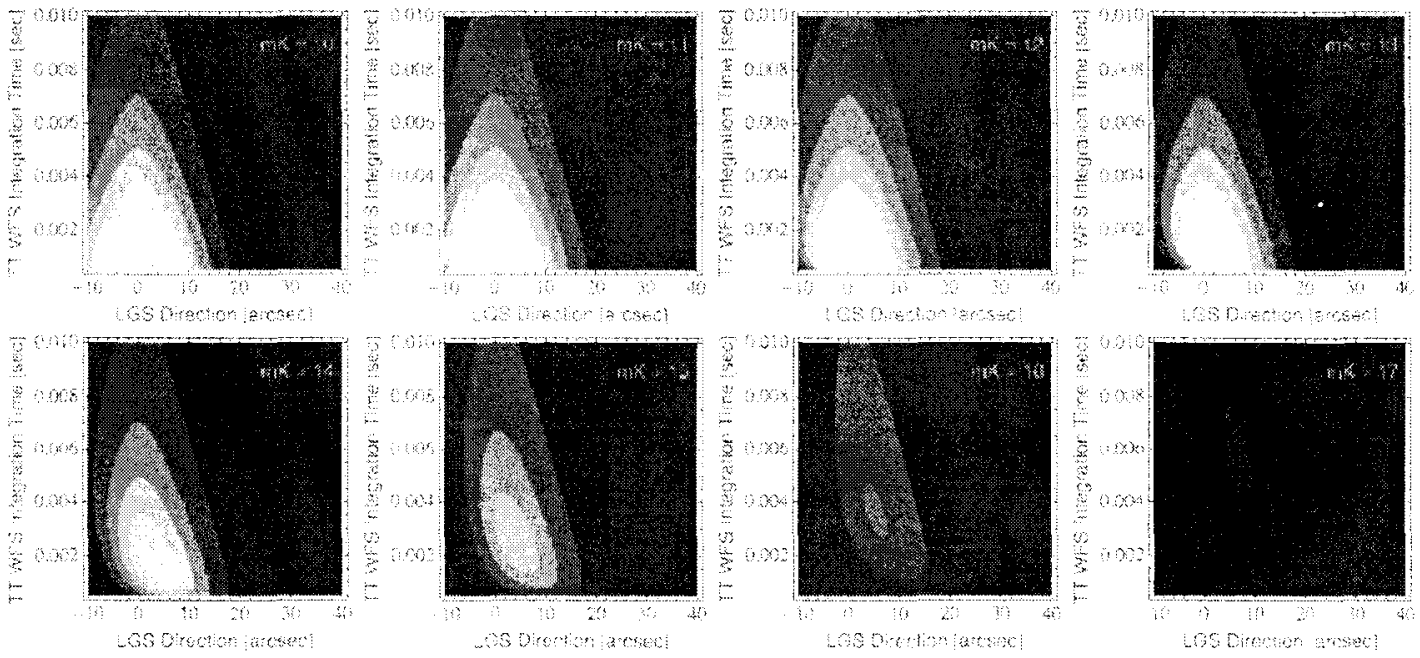

Figure 2. Science target H-band Strehl ratio performance contours for tip-tilt NGS located 30 " from the science target. having magnitudes between $10<m k<17$. Fach frane plots Strehl ratio in $2.5 \%$ contours for a range of tip-tilt sensor

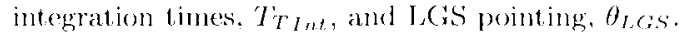

In the case of $\mathrm{K}$-band science and II-band tip-tilt wavefront sensing, the overall Strehl ratio is of course higher (Figure 3), but the trend indicates the same advantage from LGS off-pointing as the brightness of the tip-tilt NGS star decreases. At the faint linit, where science target $\mathrm{K}$-band Strehl ratio is dominated by the need for the best sharpening of the tip-tilt NGS, the optimal value of $\theta_{L G S}$ is seen to be as much as 20 areseconds in this case, or $2 / 3$ of the distance between the science target and the off-axis tip-tilt NGS.

\subsection{Potential Advantage of LGS Off-pointing}

Through optimization of both thas and ThInt, we can deternine the peak performance for Strehl ratio as a function of the tip-tilt NGS off-axis distance, $\theta$. For faint near-infrared tip-tilt NCS $\left(m_{H}=m_{K}=17\right)$, the performance comparison is shown in Figure 4. The greatest potential gain for LGS off-pointing for the scenario investigated here is seen to occur for K-band science (II-band tip-tilt sensing), when the tip-tilt NGS is off-axis by more than $\theta=15$ arcseconds. At $\theta=20$ arcseconds, the increase in $\mathrm{K}$-band Strehl ratio is dramatic, rising from $\approx 5 \%$ to nearly $20 \%$, with a corresponding determined value of $\theta_{L G S}^{O p t} \approx 10$ areseconds.

\section{ENSQUARED ENERGY PERFORMANCE WITH LGS POINTING}

Ensquared energy (EE), the fraction of light energy collected within a certain focal plane area, is more robust to residual tip-tilt errors than is Strehl ratio. Again assuming $\mathrm{K}$-band light is dedicated to tip-tilt wavefront sensing, we can compare EE performance for I-band science, as shown in Figure 5 below, with that for Strehl ratio shown in Figure 2, following the PSF assmuptions of (20) and (21). As one expects, EE is less sensitive to the exact choice of tip-tilt WFS integration time, $T_{T h n t}$, resulting to performance contours clongated in the integration time dimension. Interestingly, $O_{L G S}^{O p t}$ is reduced, by approximately $1 / 2$, compared to that determined to optimize Strehl ratio in Section 4.1. Still. I.GS off-pointing remains an important degree of freedom to optimizing science target EE, improving EE over an on-axis projection by $30 \%$ or more in the case of the faintest guide stars. 

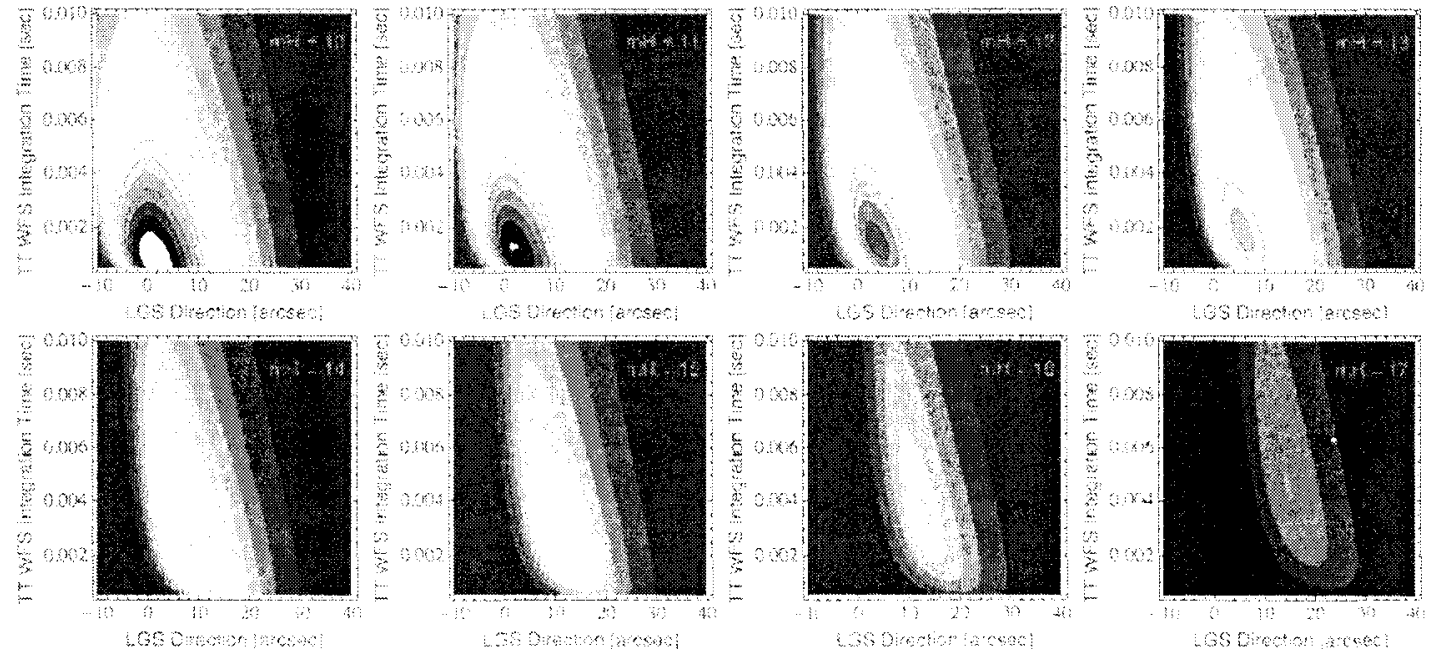

Figure 3. Science target K-band Strehl ratio performance contours for tip-tilt NGS located 30" from the science target, having magnitudes between $10<m_{K}<17$. Each frame plots Strehl ratio in $2.5 \%$ contours for a range of tip-tilt sensor integration times, $T_{T / n}$, and JCGS pointing, $\theta_{L G S}$.
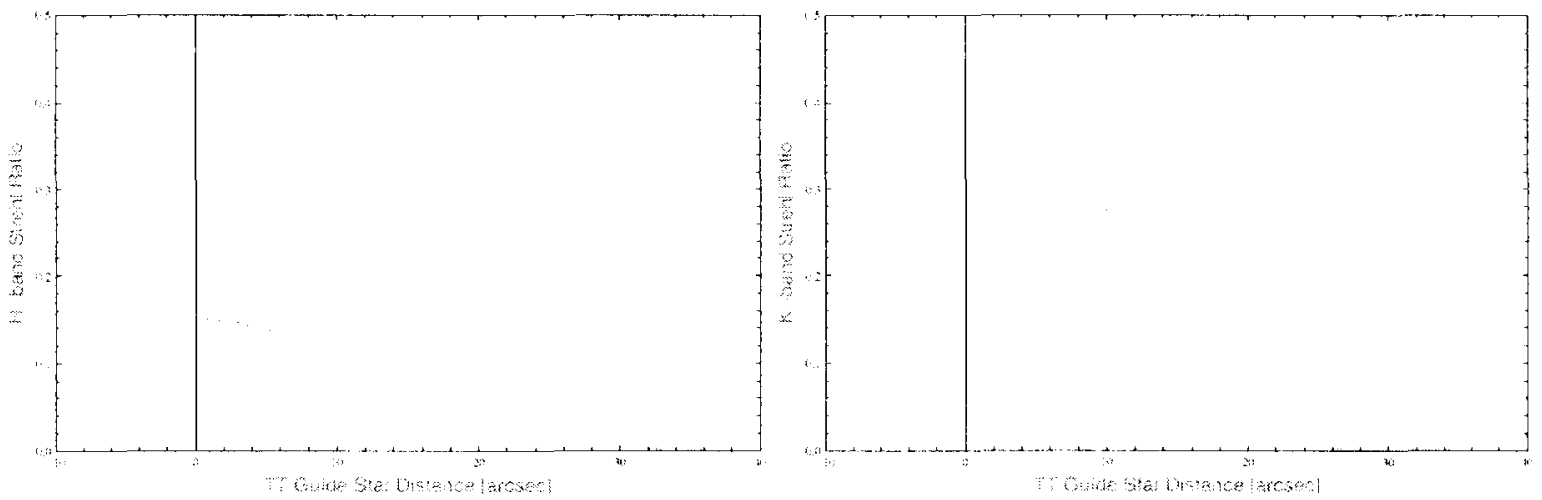

Figure 4. Science target Strehl ratio comparison for H-band (left) and K-band (right) science observations (in each case? performing tip-tilt wavefront sensing with the alternate observing band). Each figure displays the Strehl ratio obtained through optimization of $T_{T \text { Int }}$ alone for on-axis LGS pointing (solid) and through the joint optimization of $T_{T}$ Int and $\theta_{L G S}$ (dashed).

\section{CONCLUSIONS}

Arlaptive optics sharpening of tip-tilt NGS will provide signiticant increases in AO system performance metrics, such as Strehl ratio and ensquared energy. When exploiting the SNR advantage of diffraction-limited tip-tilt NGS, however, future $\Lambda O$ systems will have available (and indeed will be required to exploit) a new degree of operating freedom, namely the direction for LGS projection. As " LGS, the distance between LGS and science target is increased, angular anisoplanatism error will begin to reduce the science target Strehl attributalble to high-order wavefront errors. This effect, however, can be more than compensated by the improvement in the tip-tilt error contribution to Strehl ratio brought about through better tip-tilt NGS sharpening, particularly in the case of faint NGS. We find $\theta_{L G S}^{O p t}$ can be as large as $\theta / 2$, half the angular distance to the tip-tilt NGS. in the case of optimizing near-infrared band Strehl ratio with particularly faint NGS. For optinizing ensquared entergy, we find it more common to find $\theta_{L G S}^{O p t}=\theta / 4$, with smaller offsets in both cases when using brighter tip-tilt NGS. 

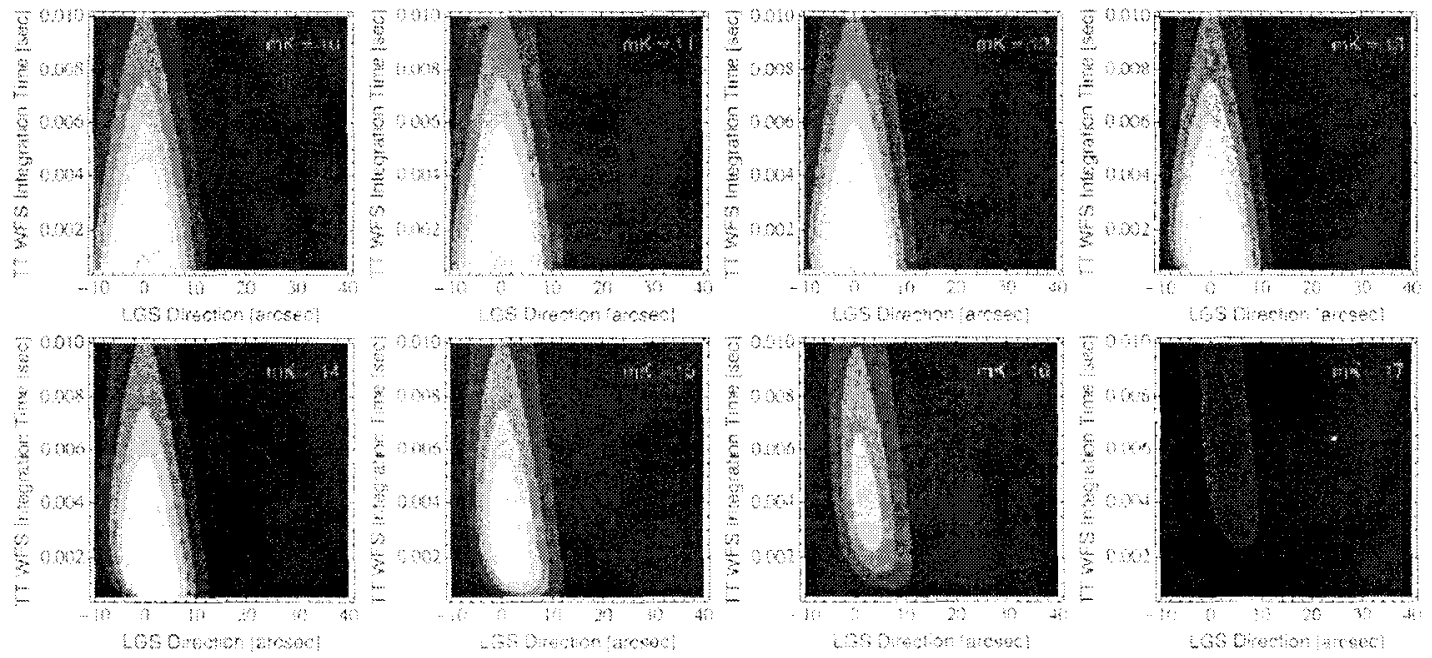

Figure 5. Science target H-band EE perfomance contours for tip-tilt NGS located 30" from the science target. having magnitudes between $10<m_{k}<17$. Each frame plots Strehl ratio in $2.5 \%$ contours for a range of tip-tilt sensor integration times, $T_{T I n t}$, and I, GS pointing, $\theta_{L C i S}$.

\section{ACKNOWLEDGMENTS}

This work has been supported by the National Science Foundation through grant AST-0619922.

\section{REFERENCES}

1. R. G. Dekany, C. Neyman, and R. Flicker, "Sharpening of natural guide stars for low-order wavefront sensing using patrolling laser guide stars," Proc. SPIE 7015, pp. 701525 701525 10, 2008

2. R. G. Dekany, B. Bamnan, D. Gavel, M. Troy, B. Macintosh, and M. Britton, "Initial concepts for CELT adaptive optics," Proc. SPIE 4839, pp. 1165-1174, 2003

3. P. L. Wizinowich, D. L. Mignant, A. H. Bouchez, R. D. Camplell, J. C. Y. Chin, A. R. Contos, M. A. van Dam, S. K. Ilartman, E. M. Johansison, R. E. Lafon, H. Lewis, P. J. Stomski, D. M. Summers, C. G. Brown, P. M. Danforth, C. E. Max, and D. M. Pemington, "The W. M. Keck Observatory laser guide star adaptive" optics system: Overview," Publications of the Astronomical Soctety of the Pacific 118. pp. 297 309, 2006.

4. P. Y. Bely, The Design and Construction of Large Optical Telescopes, Springer, New York, N.Y., 2006

5. A. Marechal Rev d'Optique 26, p. 257, 1947.

6. R. J. Sasiela and J. D. Shelton, "Transverse spectral filtering and mellin transform techniques applied to the effect of outer scale on tilt and tilt, anisoplanatism," JOSA A 10, pp. 646-660, 1993.

7. J. W. Hardy, Adaptive Optics for Astronomical Telescopes, Oxford University Press, New York, N.Y., 1998.

8. D. G. Sandler, S. Stahl, J. R. P. Angel, M. Lloyd-Hart, and D. Mec arthy. "Arlaytive optics for diffractionlimited infrared imaging with 8-m telescopes," JOSA A 11, pp. 925-945, 1994. 